\title{
Scalable and Precise Synthesis of Armchair-Edge Graphene Nanoribbon in Metal-Organic Framework
}

Takashi Kitao ${ }^{\dagger \neq}$, Michael W. A. MacLean ${ }^{\ddagger, \perp}$, Kazuki Nakata ${ }^{\ddagger}$, Masayoshi Takayanagi $^{\perp, \#, \|}$, Masataka Nagaoka ${ }^{\perp, \uparrow}$, Takashi Uemura ${ }^{\star \dagger, \ddagger, \perp}$

tDepartment of Applied Chemistry, Graduate School of Engineering, The University of Tokyo, 7-3-1 Hongo, Bunkyo-ku, Tokyo 113-8656, Japan

¥Department of Advanced Materials Science, Graduate School of Frontier Sciences, The University of Tokyo, 5-1-5 Kashiwanoha, Kashiwa, Chiba 277-8561, Japan

${ }^{\perp}$ CREST, Japan Science and Technology Agency (JST), 4-1-8 Honcho, Kawaguchi, Saitama 332-0012, Japan

\#The Center for Data Science Education and Research, Shiga University, 1-1-1 Banba, Hikone, Shiga 522-8522, Japan

"RIKEN Center for Advanced Intelligence Project, 1-4-1 Nihonbashi, Chuo-ku, Tokyo 103-0027, Japan

"Department of Complex Systems Science, Graduate School of Informatics, Nagoya University, Furo-cho, Chikusa-ku, Nagoya 464-8601, Japan 


\section{Experimental}

\section{Materials}

All reagents were purchased from commercial sources and used as received without further purification. $\mathrm{ZrO}(\mathrm{bpdc})(\mathbf{1}$; bpdc = biphenyl-4,4'-dicarboxylate) was prepared according to previously described methods. ${ }^{1}$ Dibromoperylene (BrPER) was synthesized according to previously reported in the literature. ${ }^{2}$

\section{Polymerization of perylene (PER) within 1}

PER was finely ground, and mixed with the degassed $1(250 \mathrm{mg})$. The resulting mixture of 1 and PER was heated at $180^{\circ} \mathrm{C}$ for $1 \mathrm{~h}$ under reduced pressure $(<0.3 \mathrm{kPa})$, leading to vapor adsorption of PER throughout the internal and external surface of 1 . The externally adsorbed monomer was selectively removed by heating at $180{ }^{\circ} \mathrm{C}$ for $3 \mathrm{~h}$ under vacuum,

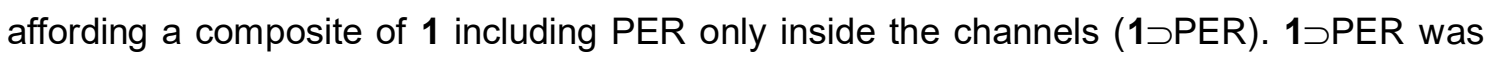
subjected to TG analysis to determine the loading amount of PER. 1つPER was heated at $400{ }^{\circ} \mathrm{C}$ (BrPER: $300{ }^{\circ} \mathrm{C}$ ) for $24 \mathrm{~h}$ in a sealed glass container, affording 1-armchair GNR composite (1つAGNR). Upon cooling, the solid was washed with DMF $(100 \mathrm{ml})$ to remove the unreacted monomer.

\section{Isolation of AGNR from 1}

$1 \supset$ AGNR (116 mg) was stirred for 2 days in a $0.05 \mathrm{M}$ aqueous solution of sodium ethylenediaminetetraacetate $(40 \mathrm{ml})$, followed by washing treatment with $5 \% \mathrm{v} / \mathrm{v}$ aqueous solution of HF (4 ml) for the dissolution of the framework of 1, yielding AGNR (8 mg, $28 \%$ conversion). When BrPER was used as a monomer, $48 \mathrm{mg}$ of AGNR was obtained from 540 mg of $1 \supset$ AGNR (54\% conversion). 


\section{Physical measurements}

X-ray powder diffraction (XRPD) data were collected on a Rigaku SmartLab Diffractometer using a $\mathrm{Cu}$ anode and a $\mathrm{K} \alpha$ monochromator $(\lambda=0.154 \mathrm{~nm})$. Solid-state NMR measurements were carried out on a 9.4 T Bruker solid-state NMR instrument with an ADVANCE III $400 \mathrm{MHz}$ spectrometer. ${ }^{13} \mathrm{C}$ cross-polarization magic-angle spinning NMR spectra were obtained using a double resonance $4 \mathrm{~mm}$ magic-angle spinning probe, with recycle delay and spinning rate of $2 \mathrm{~s}$ and $12 \mathrm{kHz}$, respectively. The adsorption isotherms of $\mathrm{N}_{2}$ at $77 \mathrm{~K}$ were measured using BELSORP-mini equipment. Before the adsorption measurements, the sample was treated under reduced pressure $\left(<10^{-2} \mathrm{~Pa}\right)$ at $373 \mathrm{~K}$ for $5 \mathrm{~h}$. Matrix-assisted laser desorption/ionization time-of-flight mass spectrometry (MALDI-TOF-MS) spectra were recorded on an AB Sciex TOF/TOF 5800 using trans-2-[3-(4-tert-buthylphenyl)-2-methyl-2-propenylidene]malononitrile as the matrix. Scanning electron microscopy-energy dispersive X-ray (SEM-EDX) measurements were conducted using a HORIBA EMAXEvolution EX-370 attached to a HITACHI S-3000N operated at an accelerating voltage of $20 \mathrm{kV}$. Samples were placed on a conducting carbon tape attached by SEM grid, and then coated with platinum. Thermogravimetric (TG) analysis was conducted from room temperature to $500{ }^{\circ} \mathrm{C}$ at $10 \mathrm{~K} \mathrm{~min}^{-1}$ using a Rigaku Instrument Thermo plus TG 8120 in an atmosphere of nitrogen. Particle size distribution was obtained by Horiba Partica LA-950 laser diffraction particle size analyser. Microscopic Raman spectroscopy was carried out using JASCO NRS-4500. $532 \mathrm{~nm}$ and $785 \mathrm{~nm}$ semiconductor lasers were used as the excitation source. IR spectra were measured using a JASCO FT/IR-4200. UV-vis spectra were recorded using a JASCO V-670 spectrophotometer.

\section{Molecular dynamics (MD) simulations}

All the MD simulations were executed by the sander module of the Amber14 package. A simplified model of $1 \supset$ PER was prepared as a single one-dimensional (1-D) channel of 1 containing 5 guest PER molecules. The channel was only composed of bpdc ligands, whose oxygen atoms were fixed at the initial positions to keep the channel structure. The 5 PER molecules were then enclosed in the channel and both terminal ones were fixed at the initial positions to trap the remaining 3 PER molecules in the channel. The force field parameters were retrieved from the general Amber force field. The atomic charges were assigned by the Merz-Kollman scheme with density functional theory (DFT) calculations at the level of M06-2X/6-31+G(d) using the Gaussian09 package. For the bpdc ligand, the force field parameters for the dihedral angle rotation of the benzene rings were optimized to reproduce rotational barriers obtained by DFT calculations. In addition, the atomic charges were calculated by a $\mathrm{Li}^{+}$-capped model to neutralize the carboxylate groups, and the atomic 
charge of $\mathrm{Li}^{+}$was distributed to the oxygen atoms. The integration time step was $2 \mathrm{fs}$ and the SHAKE algorithm was utilized to freeze bonds involving hydrogen atoms. The temperature was kept at $673 \mathrm{~K}$ by the weak-coupling algorism. Finally, we executed $1 \mathrm{~ns}$ MD simulation. 


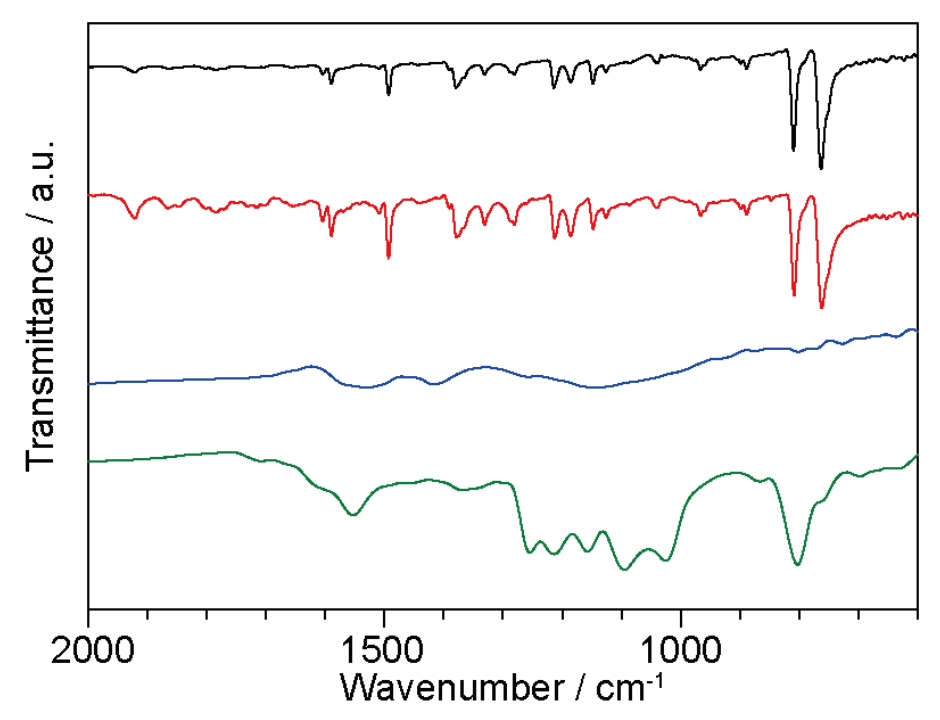

Figure S1. IR spectra of PER (black), PER after heating treatment at $400{ }^{\circ} \mathrm{C}$ (red) and $600{ }^{\circ} \mathrm{C}$ (blue), and AGNR liberated from 1 (green). The identical IR spectra indicated that no reaction proceeded at $400{ }^{\circ} \mathrm{C}$. After heating at $600{ }^{\circ} \mathrm{C}$, the peaks located in the 750 $850 \mathrm{~cm}^{-1}$, corresponding to out-of-plane $\mathrm{sp}^{2} \mathrm{C}-\mathrm{H}$ vibration modes, completely disappeared. This result suggested the formation of branched or graphitic carbon via dehydrogenative condensation reaction. ${ }^{3}$ In contrast, AGNR liberated from 1 showed the clear peaks in the same wavenumber regions. The relative intensity of the peak at 808 $\mathrm{cm}^{-1}$ to $762 \mathrm{~cm}^{-1}$ increased after the formation of AGNR. Because the peak at $808 \mathrm{~cm}^{-1}$ are assignable to the $\mathrm{sp}^{2} \mathrm{C}-\mathrm{H}$ vibration mode at armchair edge, this result was suggestive of the growth of the nanoribbon while maintaining the armchair edge structure. ${ }^{4,5}$

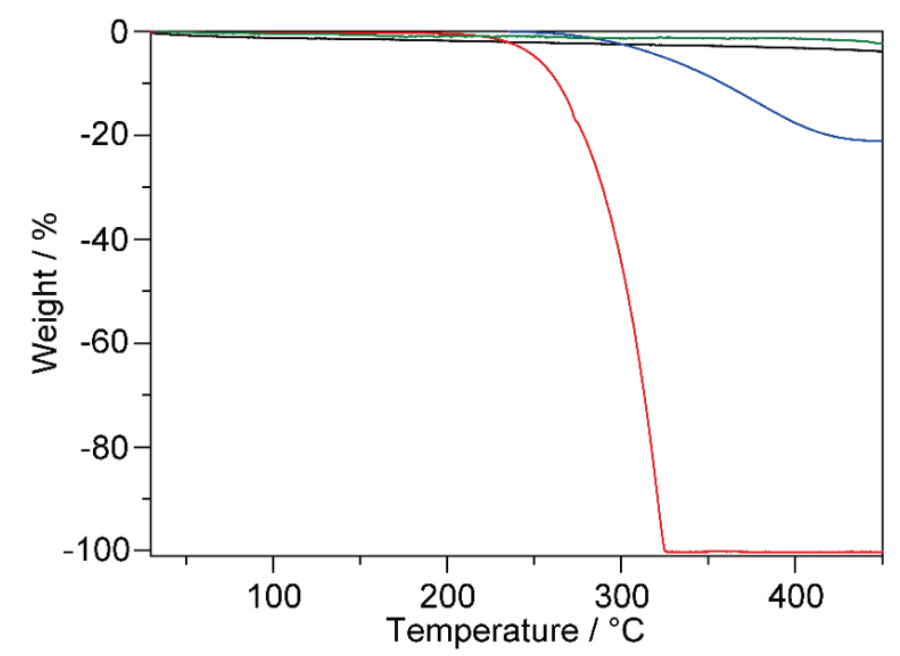

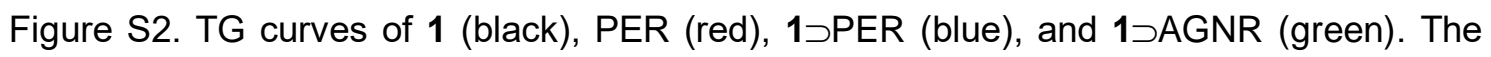
monomer was completely removed by washing treatment, as confirmed by the lack of weight loss of PER in TG curve of $1 \supset A G N R$. 


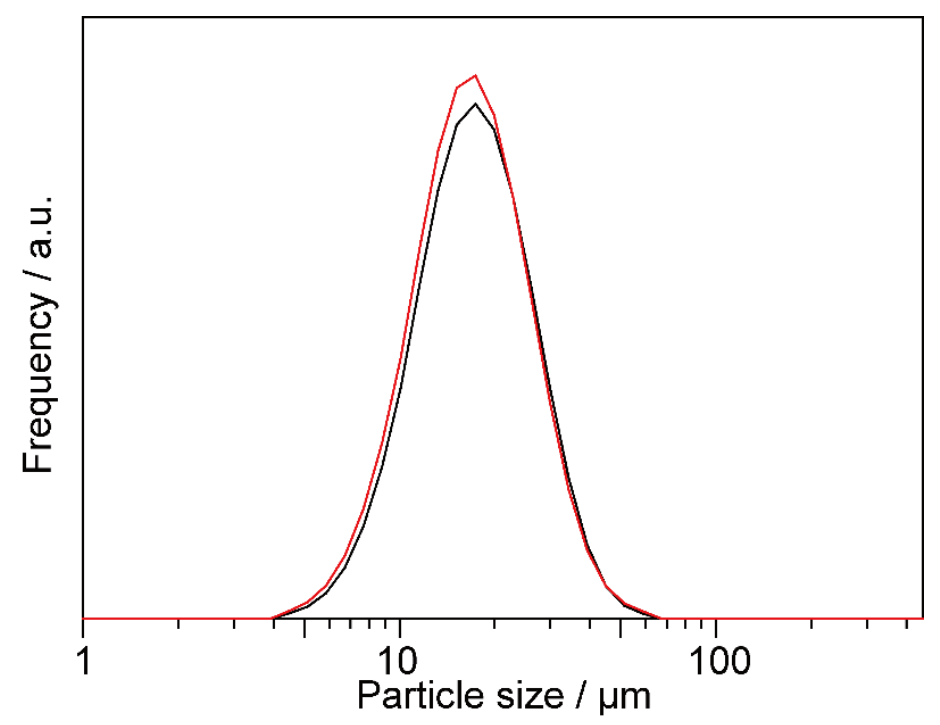

Figure S3. Particle size distributions of 1 (black) and $1 \supset$ AGNR (red) evaluated by laser light diffraction. The size distribution of $1 \supset A G N R$ was almost the same to that of 1 , indicating no AGNR existing outside 1.
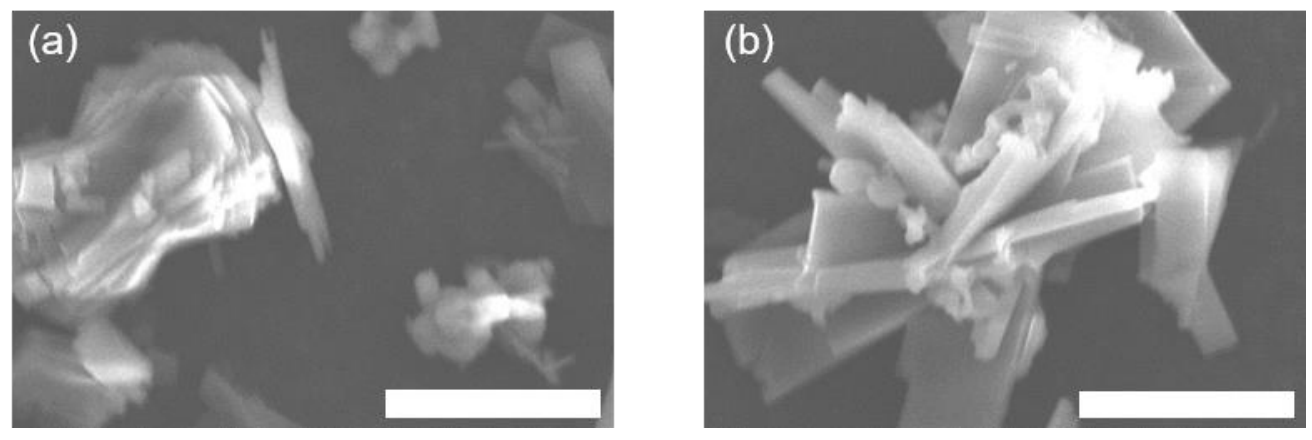

Figure S4. SEM images of (a) 1 and (b) $1 \supset$ AGNR. Scale bar $=10 \mu \mathrm{m}$. Morphology of the host particles in SEM images was completely the same before and after the polymerization, showing that the polymerization proceeded only within the nanochannels. 


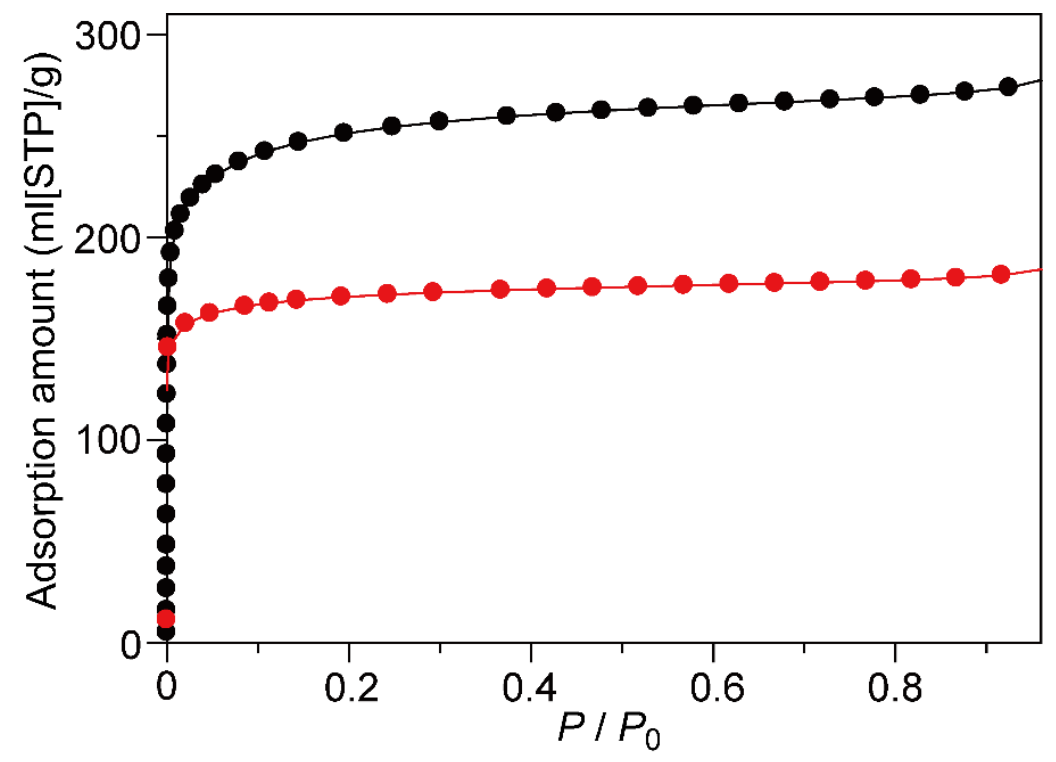

Figure S5. $\mathrm{N}_{2}$ adsorption isotherms of 1 (black) and $1 \supset$ AGNR (red) at $77 \mathrm{~K}$. The adsorption isotherm of $1 \supset A G N R$ showed decrease in the amount of adsorption compared with 1, which clearly indicated the presence of AGNR within the nanochannels.
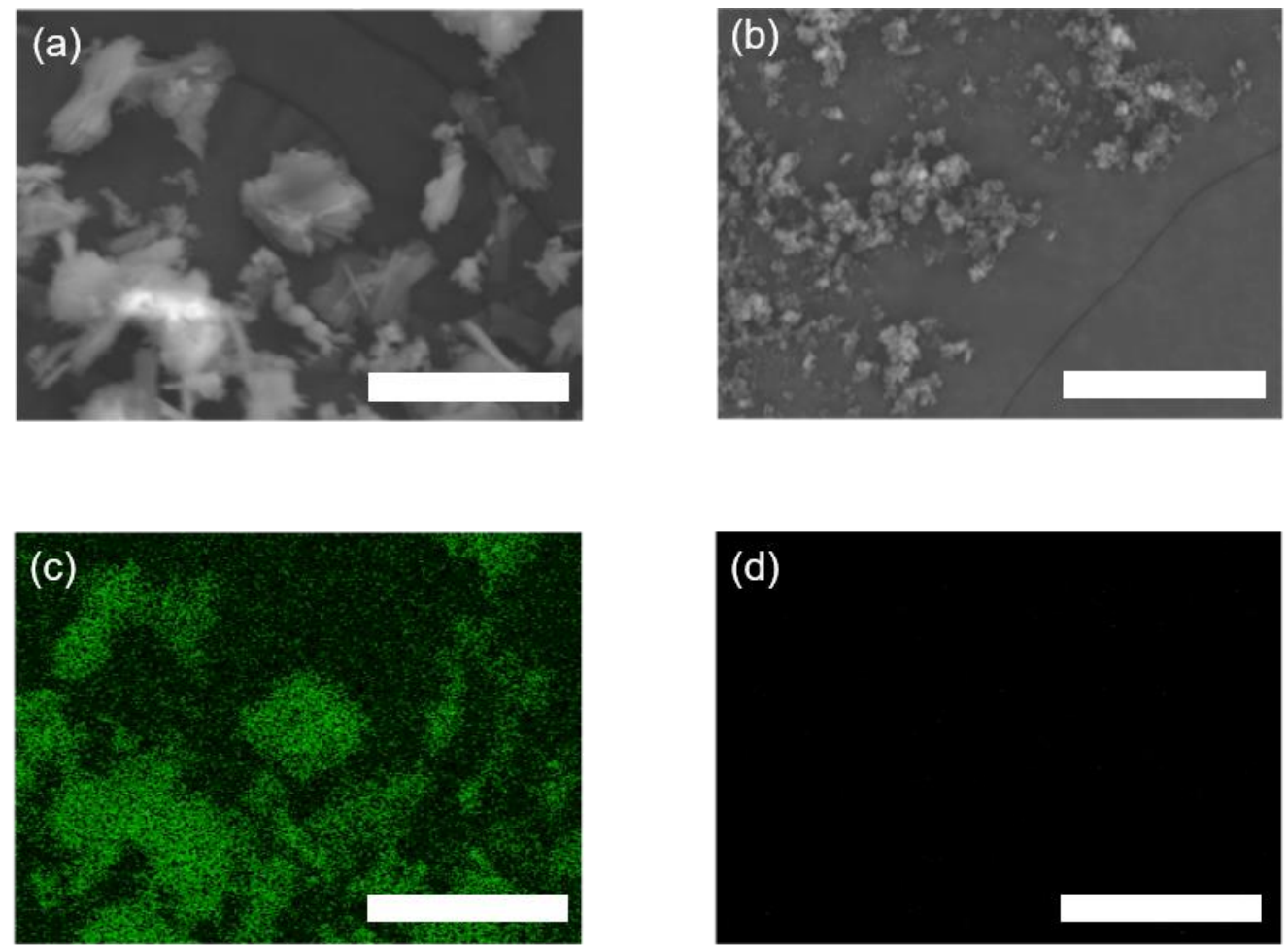

Figure S6. SEM images of (a) 1つAGNR and (b) AGNR liberated from 1. EDX analysis (Zr mapping) of the corresponding (c) $1 \supset A G N R$ and (d) AGNR, which demonstrates the complete removal of 1 during the GNR recovery process. Scale bar $=25 \mu \mathrm{m}$. 


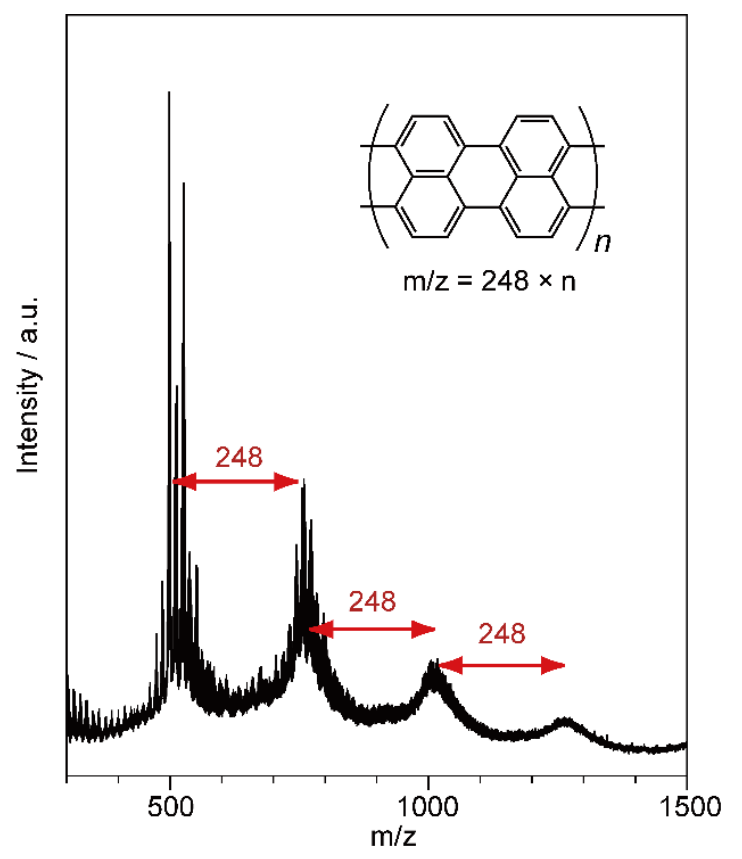

Figure S7. MALDI-TOF-MS spectrum of AGNR liberated from 1. MALDI-TOF-MS spectra displayed a repeating mass interval, corresponding to the monomer units, which confirmed formation of AGNR through the coupling reaction of the monomer. The broadening of the peaks was observed, which would be attributed to low ionization efficiency of AGNR. ${ }^{6}$

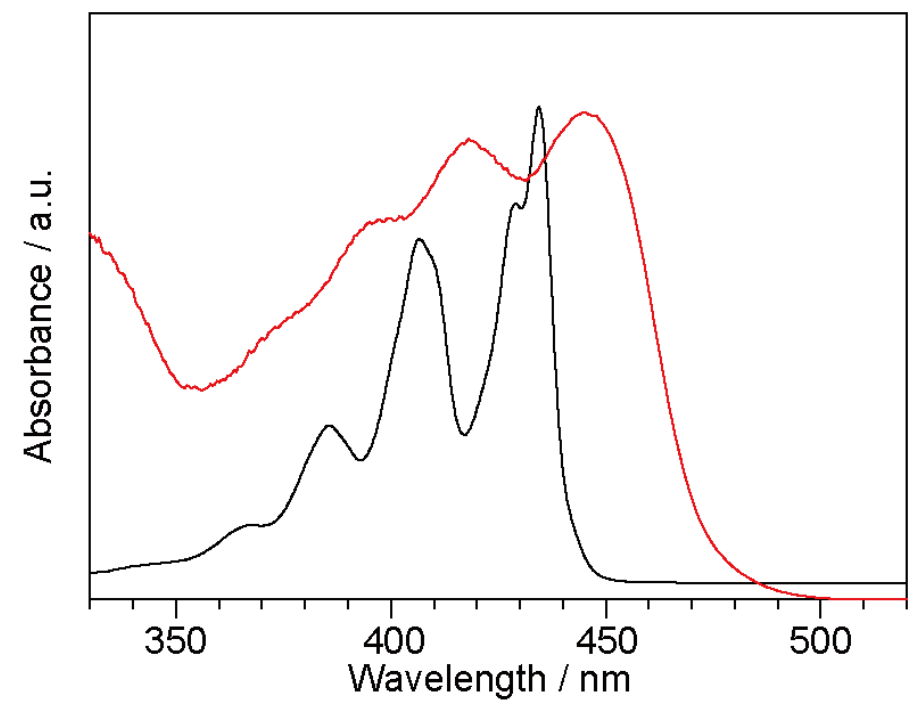

Figure S8. UV/vis spectra of the PER hexane solution (black) and 1دPER (red). The absorption band of $1 \supset$ PER redshifted against PER in solution. This shift would be the consequence of the formation of $\mathrm{J}$-aggregates of PER within the 1-D nanochannels. ${ }^{7}$ 


\section{REFERENCES}

1. Guillerm, V.; Ragon, F.; Dan-Hardi, M.; Devic, T.; Vishnuvarthan, M.; Campo, B.; Vimont, A.; Clet, G.; Yang, Q.; Maurin, G.; Férey, G.; Vittadini, A.; Gross, S.; Serre, C. A Series of Isoreticular, Highly Stable, Porous Zirconium Oxide Based Metal-Organic Frameworks. Angew. Chem. Int. Ed. 2012, 51, 9267-9271.

2. Sakaguchi, H.; Kawagoe, Y.; Hirano, Y.; Iruka, T.; Yano, M.; Nakae, T. Width-Controlled Sub-Nanometer Graphene Nanoribbon Films Synthesized by Radical-Polymerized Chemical Vapor Deposition. Adv. Mater. 2014, 26, 4134-4138.

3. Biniak, S.; Pakuła, M.; Darlewski, W.; Świątkowski, A.; Kula, P. Powdered activated carbon and carbon paste electrodes: comparison of electrochemical behaviour. J. Appl. Electrochem. 2009, 39, 593-600.

4. Centrone, A.; Brambilla, L.; Renouard, T.; Gherghel, L.; Mathis, C.; Müllen, K.; Zerbi, G. Structure of new carbonaceous materials: The role of vibrational spectroscopy. Carbon 2005, 43, 1593-1609.

5. Sasaki, T.; Yamada, Y.; Sato, S. Quantitative Analysis of Zigzag and Armchair Edges on Carbon Materials with and without Pentagons Using Infrared Spectroscopy. Anal. Chem. 2018, 90, 10724-10731.

6. Veber, G.; Diercks, C. S.; Rogers, C.; Perkins, W. S.; Ciston, J.; Lee, K.; Llinas, J. P.; Liebman-Peláez, A.; Zhu, C.; Bokor, J.; Fischer, F. R. Reticular Growth of Graphene Nanoribbon 2D Covalent Organic Frameworks. Chem 2020, 6, 1-9.

7. Herbst, S.; Soberats, B.; Leowanawat, P.; Stolte, M.; Lehmann, M.; Würthner, F. Self-assembly of multi-stranded perylene dye J-aggregates in columnar liquid-crystalline phases. Nat. Commun. 2018, 9, 2646. 